\title{
Improving the Safety of Aging Road Users: A Mini-Review
}

\author{
Walter R. Boot Cary Stothart Neil Charness \\ Department of Psychology, Florida State University, Tallahassee, Fla., USA
}

\section{Key Words}

Transportation $\cdot$ Safety $\cdot$ Countermeasures $\cdot$ Driver training

\begin{abstract}
Older drivers are at greatest risk for injury or death as a result of a car crash. In this mini-review, we outline the normative age-related changes to perceptual, cognitive, and motor abilities that contribute to increased crash risk and decreased comfort with driving, and highlight specific driving scenarios and conditions that are particularly challenging for aging road users. Adopting a person-environment fit framework, we discuss how the roadway environment can be modified to better match the abilities of the aging driver. We also review evidence for the efficacy of training interventions that aim to change the abilities and strategies of the aging driver to better match the demands of the driving environment. Evidence suggests that specific changes to the roadway and driver training strategies can bring the abilities of the older driver back into alignment with the demands of the driving task. A focus on both approaches will help ensure the safety of all road users as the number of aging drivers greatly increases over the next few decades.
\end{abstract}

(c) 2013 S. Karger AG, Basel (c) 2013 S. Karger AG, Basel

0304-324X/14/0601-0090\$39.50/0

\section{Introduction}

Per mile driven, older drivers ( $\geq 65$ years of age) have greater crash and fatality rates compared to any other age group except for the most inexperienced drivers $(\leq 25$ years of age). This trend is largely driven by an accelerating crash risk after the age of 70 years coupled with greater physical frailty in old age with respect to crash forces [1]. Addressing issues related to aging road user safety is becoming increasingly important as the United States and other industrialized nations undergo the process of population aging. By the year 2050 the proportion of the population made up of older adults will double, and with the aging of the 'baby boomer' generation we can expect a substantial increase in the number of older drivers on the road.

While driving cessation (self-imposed or mandated) might seem like an acceptable solution to improving the safety of road users, it is important to recognize that the potential benefits are also associated with substantial costs. First, driving cessation places the burden of transportation on family and caregivers and may be especially difficult in rural communities lacking adequate public transportation. Second, driving cessation is associated with a number of

\section{KARGER}

E-Mail karger@karger.com

www.karger.com/ger
Walter R. Boot, $\mathrm{PhD}$

Department of Psychology

Florida State University, 1107 W. Call Street

Tallahassee, FL 32306-4301 (USA)

E-Mail boot@psy.fsu.edu 
negative outcomes including increased risk of depression, isolation, and a decreased quality of life and health (e.g. [2]). Since many older adults wish to continue to drive to maintain their independence, and because there are a number of negative outcomes associated with driving cessation, this review primarily focuses on alternative means to improving road users' safety and comfort.

In discussing age-related driving problems and potential solutions, we adopt a person-environment fit framework. Perceptual and cognitive abilities decline with age, making aspects of the driving task more challenging for older drivers compared to younger drivers (although greater experience may offset these difficulties to some extent). There are two general approaches to improving driver safety within this framework: (1) change the abilities or strategies of the person, or (2) change the characteristics of the environment. The goals of this mini-review are to describe the perceptual and cognitive declines associated with increasing age that potentially play a role in road users' comfort and safety, discuss how these declines impact specific high-risk driving situations, review changes to the road environment that support the safety and comfort of aging drivers, and review the efficacy of older driver training programs in improving driving performance and reducing crash risk.

\section{Driving-Relevant Age-Related Perceptual and Cognitive Changes}

A recent report found that seniors were disproportionately involved in crashes while making a turn across opposing traffic at intersections, merging from a yield lane, or changing lanes on a highway [3]. Numerous complex perceptual and cognitive processes are involved in situations in which a driver must decide when it is safe to enter a moving stream of traffic. We outline some of the normative age-related changes in vision, hearing, cognition, and psychomotor speed that provide critical information for these and other driving decisions (see [4] for an in-depth treatment of age-related perceptual/cognitive decline and its impact on performance). However, we caution that direct links between age-related changes and relatively rare crashes are difficult to establish because age-related declines in one sensory system may be correlated with declines in others. Further, because sensory system acuity is also strongly related to measures of cognition in adulthood, it is challenging to unpack relationships between perceptual and cognitive decline and driver comfort and safety outcomes.

Safety and Aging Road Users

\section{Vision}

The driving task is primarily visual in nature, and impaired vision is associated with increased driver discomfort, difficulty, and crash risk [5]. The visual system and visual perception change in a number of important ways with increasing age [6], and these changes have implications for safe driving. For example, most people in their 40 s lose the ability to focus their eyes for near vision (presbyopia) due primarily to thickening of the lens. Presbyopia might affect someone's ability to process visual information as they shift focus from monitoring the road ahead to monitoring the instrument panel. Decreased maximal pupillary dilation, coupled with yellowing of the lens and poorer transmission of light through the optical media (e.g. from cataract formation) result in a substantial reduction in the amount of light reaching the retina. A 65-year-old eye may admit only one third of the light of a 20 -year-old eye under low light conditions. These changes, coupled with impaired neural processes responsible for dark and light adaptation, make night driving particularly challenging for older drivers. Changes in depth perception may make it difficult to judge gaps in traffic accurately, leading to poorer merge and turn decisions. Furthermore, eye diseases that increase sharply in late adulthood such as cataracts, glaucoma, diabetic retinopathy, and macular degeneration can seriously impair visual acuity.

\section{Hearing and Vibration Detection}

Although driving is primarily a visually guided task, hearing is also a useful perceptual channel (e.g. hearing a horn warning from a fellow motorist, a siren from an unseen emergency vehicle, or the audible click feedback of a lane-change signal). The processing of potentially important driving-relevant auditory signals may be impaired because hearing declines markedly as age increases [7], with hearing loss accelerating past the age of 60 . This is particularly true for men, and mainly in the higher frequency ranges $(2,000 \mathrm{~Hz}$ and higher). The ability to process signals in the presence of noise has also shown a strong age-related decline. With respect to driving performance, self-reported hearing loss is a risk factor for crashes [8], and not surprisingly, hearing impairment may be especially problematic when accompanied by vision issues [9]. Although modern vehicle designs have benefited from noise reduction techniques, there is the possibility for road noise to mask relevant signals. Vibration detection, particularly for high frequencies, also declines with age. Thus, even multimodal sound and vibration signals created by raised center lane markers and 
lane rumble strips might be more difficult to detect quickly, potentially reducing their value for correcting lane drift.

\section{Attention}

One of the requirements for safe driving is the ability to scan the visual field for relevant objects and take appropriate action. High acuity vision is limited to a few degrees of the visual angle (the width of your thumb held out at arm's length). Thus, it is necessary to scan the visual field by making eye movements or head movements to search for relevant information. Visual search efficiency declines with age, particularly in terms of being able to prevent attention from returning to already searched locations (inhibition of return), with age-related declines being linked to driving performance measures [10]. In addition, a driver's ability to divide his/her attention between a central location and the periphery shows a much sharper decline with age. Such shrinkage in the useful field of view [11] is a strong predictor of crash rates [12]. The ability to rapidly switch one's attention also declines markedly with age, i.e. an older driver may be slower to switch his/her attention from one aspect of the driving task to another. An example would be looking ahead to judge the distance to a followed vehicle and then switching attention to the side to detect a vehicle merging into traffic from an on-ramp.

\section{Speed of Processing and Responding}

A hallmark of aging is decline in the speed of processing [13]. Older adults take 1.7-2.0 times longer than younger adults for elementary information processing operations (perceptual, cognitive, and motor processing [14]). Such slowing has implications for driving both in terms of adaptations that are necessary for older adults to drive safely and for how to design roadways to provide warnings early enough to allow safe decisions (e.g. road exit signs). In general, skilled driving requires that drivers anticipate hazardous conditions rather than relying on quick reflexes to react to them.

\section{Disease Processes}

As people age, they are likely to incur chronic conditions such as heart disease, cancer, diabetes, and dementia. By age $65,90 \%$ of the population are likely to have one or more chronic conditions [15]. These conditions, coupled with the medications taken to control them, have the potential to impair functioning. For example, diabetic drivers are 1.5 times more likely to be involved in an atfault crash, and individuals with cognitive impairment are 3 times as likely to be involved in an at-fault crash [16] (see [17] for an example of counterevidence). Arthritis can also make it more difficult to perform actions important for safe driving, including the act of turning one's head to check blind spots.

\section{Accommodating Age-Related Changes}

In many cases, older adults can adapt to the discussed changes, allowing them to continue to drive safely. One potential adaptation involves self-regulation. Older drivers tend to avoid challenging driving conditions (night driving, poor weather) and often drive at reduced speeds to provide themselves with increased time to react to changing traffic conditions (although see evidence reported later that self-regulation alone may not decrease crash risk). Another way to help older drivers is to design better vehicles and road systems that make fewer demands on waning abilities. To summarize, most of the changes described above can be expected to affect older adult drivers; these are changes that are a normal part of the aging process. They result in a mismatch between the demands of the driving task and the abilities of the driver. Next we turn to specific roadway challenges that arise from this mismatch.

\section{Age-Related Driving Difficulties}

\section{The Challenge of Turning across Opposing Traffic}

Even though intersections account for a small proportion of the total roadway, a disproportionate number of traffic-related fatalities and injuries occur at or near intersections. Crashes at intersections in which a vehicle is struck while turning to cross opposing traffic (left-turn crashes in countries that drive on the right side of the road, right-turn crashes in countries that drive on the left side of the road) are both common and severe. Older adult drivers are at greater risk for intersection crashes, and crashes in which a turning vehicle is struck by opposing traffic are particularly dangerous and common for older adult drivers [18]. Differential risk can be linked to difficulties with speed and gap judgments [19], as well as decreased working memory capacity [20].

\section{Nighttime Driving}

On a per-mile basis, fatal crashes are more likely to occur at night. Although older adults are less likely to be involved in nighttime crashes compared to younger adults, infrequent nighttime crashes may be the result of a strategy of avoiding nighttime driving. Older adults
Boot/Stothart/Charness 
who do drive at night are at greater risk due to age-related changes in the amount of light reaching the retina, poorer contrast sensitivity, and changes in dark and light adaptation. Steering accuracy, for example, appears to be differentially impaired for older adults compared to younger adults under conditions of low illumination [21].

\section{Road-Hazard Detection}

A critical component of the driving task is the ability to anticipate, detect, and respond to changes in roadway conditions. Age-related perceptual and cognitive declines can decrease the speed and accuracy of doing so. For example, Caird et al. [22] presented younger and older participants with images of intersections and had participants make decisions about whether or not it would be safe to proceed through an intersection, turn left, or turn right. During the presentation, cars or pedestrians were sometimes inserted in the path of intended travel. Older adults were less likely to detect these hazards (i.e. they were more likely to judge that it was safe to proceed), and were particularly less likely to detect the presence of pedestrians.

\section{Sign Perception}

Signs and signals convey important information critical for safe driving and efficient navigation. Previously described changes to the visual system can influence older adults' ability to notice and comprehend the meaning of signs. For instance, Dewar et al. [23] found that older adult participants tended to display legibility distances that were $80 \%$ of what their younger counterparts displayed. In addition, they found that glare reduced legibility distances, but only for the older adult participants. Critical information conveyed by signs and signals may not reach older drivers quickly enough, putting them at greater risk compared to younger drivers.

\section{Countermeasures to Improve Road User Safety}

Within the person-environment fit framework, one option to reduce crashes is to change the environment to better match the abilities of the driver. While these changes might be especially important in reducing agerelated crash risk, they also make the driving task easier for drivers of all ages. Specific countermeasures are described below (see Staplin et al. [24] for additional recommendations and justification for proposed roadway changes).

Safety and Aging Road Users

\section{Offset Turn Lanes}

Crashes involving a turning car being struck by oncoming traffic are common and older adults are at a greater risk for this type of crash. One potentially effective countermeasure to support older drivers is the use of offset turn lanes. For example, in the USA an opposing left-turn lane can be offset to the right to allow a more unobstructed view of oncoming traffic when the opposing left-turn lane is occupied by vehicles. This design aids older drivers who tend to have an impaired ability to correctly judge speed/distances and gaps between vehicles by giving them more time to estimate these values and make a more accurate decision regarding when to turn [25]. As suggested by Preusser et al. [18], a protected turn phase may also be beneficial to older drivers. Protected turn signals greatly simplify the decision process involved when turning across traffic, but may come at a cost in terms of traffic flow.

\section{Improving Nighttime Visibility}

A number of countermeasures have the potential to address the difficulties older drivers face when driving at night. These include internally lit street signs, improved roadway illumination [26], highly reflective (fluorescent) backing on signs, and raised reflective pavement markers that more easily delineate the roadway even under lowlight conditions.

\section{Advanced Street Name Signs}

Advanced street name signs (street name signs placed a certain distance before a roadway) are another potentially effective countermeasure for older adults. These signs are beneficial in that they give older drivers an earlier opportunity to identify an upcoming road, and as a result reduce their risk of making a rear-end or side-swipe collision that may result from decreased attention to the road while attempting to read street signs at intersections. Gross et al. [27] analyzed data from 193 signalized intersections before and after the placement of advanced street name signs and observed that side-swipe collisions were reduced by up to $27 \%$.

\section{Increased Text Size}

Text is generally easier to read when it is larger. Therefore, increasing the character size of roadside signs can result in increased readability, especially for older adults who are more likely to suffer from degraded perceptual abilities. In an older adult sample, Guerrier and Fu [28] observed a benefit from larger font sizes in terms of increased legibility distances (older adults were able to read signs from a greater distance when the font size was larg- 
er). Similar to advanced street name signs, greater sign legibility distances give older adults more time to prepare a response.

\section{Modifying Perception-Action Time Estimates of Older} Drivers

Roadway design and sign and signal placement are based on the time estimated for drivers to be able to perceive and respond to events and messages, and these estimates may not adequately account for age-related slowing. For example, when estimating the appropriate duration of a yellow light, a parameter specifying the drivers' perception-response time must be taken into account. This parameter is $1 \mathrm{~s}$ according to the Manual on Uniform Traffic Control Devices, which may be too short for older drivers. By using GOMS modeling [14, 29], it was found that older adults take $20-100 \%$ longer to process information and respond compared to younger adults. Therefore, it may be beneficial to adjust the normal time interval on yellow lights accordingly. Similar modeling efforts could be used to improve other estimates used to make decisions on roadway design. However, longer perception-reaction times may be offset by the tendency of older drivers to drive more slowly [30].

\section{Aging Road User Training}

In addition to various changes that can be made to the roadway to reduce the crash risk of older drivers, the person-environment fit model also suggests that we can improve the abilities of older drivers to help them cope with the demands of driving despite age-related changes that make driving more challenging. Many studies have examined the effect of various interventions on driving knowledge, performance (either on road tests or in simulators), and to a lesser extent crash risk. While there has been success in improving older adults' knowledge, use of strategy, and driving performance in certain situations, the evidence that these improvements result in decreased crash rates is unfortunately limited.

\section{Older Driver Education Programs}

Older driver education has been a focus of a number of interventions intent on encouraging safe driving and reducing crash risk. These programs are designed to increase awareness of age-related risk, highlight specific driving conditions and situations that are most hazardous to older drivers, and encourage strategies for coping with or avoiding these risky driving conditions. For example, Owsley et al. [31] educated drivers suffering from age-related visual decline on the impact of poor vision on driving safety and specific strategies to decrease crash risk [e.g. driving only during the day, making three right turns (in the USA) instead of a left turn at a busy intersection, etc.]. This intervention was effective in increasing self-reported strategy use and improving awareness of driving difficulty and decreased visual ability, but unfortunately was not associated with a decreased crash rate 2 years after the intervention. Similar null results with respect to crash rate were reported by Janke [32] after evaluating the impact of a driver education program, and at least one study has found an increased crash rate for older drivers who participated in a driver education program compared to older drivers who did not. In general, education programs appear to be effective at increasing awareness and reducing exposure to risky driving situations, but there is limited evidence that driver education alone can reduce crash rates.

\section{Education plus On-Road Training}

Recent studies have evaluated the impact of driver education programs combined with on-road driver training, and these multipronged interventions have indicated promise. For example, participants assigned to the intervention condition in a study conducted by Bédard [33] completed an educational component based on the program developed by the AARP, but also received two road sessions with a driving instructor that reinforced the concepts presented in this program. The intervention group demonstrated greater knowledge of safe driving practices after the intervention compared to participants who did not receive the intervention, but also were evaluated as making fewer unsafe roadway maneuvers in a driving test. Similar results were observed by Marottoli et al. [34]. However, these studies did not evaluate whether changes in driving behavior result in fewer crashes after the intervention.

\section{Perceptual Training}

As mentioned previously, visual processing speed, often measured by the useful field of view, declines with age and this decline is predictive of crashes. Based on the known relationship between visual processing speed, age, and driver safety, some interventions focus on training visual abilities to reduce the crash risk of older drivers. For example, Roenker et al. [35] conducted a study in which participants received driving simulator training or useful field of view training. Driving performance was then evaluated by an on-road examination. Participants who received visual processing speed training engaged in fewer risky maneuvers. Furthermore, in a driving simulator-based reac- 
tion time task, visual processing speed training was associated with reduced reaction times. However, this was a relatively small study in terms of sample size of the intervention and control group, and crash rates were not assessed. As part of the ACTIVE (Advanced Cognitive Training for Independent and Vital Elderly) clinical trial, a similar training intervention was compared to two active and one passive control group ( $\mathrm{n}=908$ in total), and crashes were recorded for 6 years [36]. Although the total crash rate did not differ between the groups, the processing speed-trained group was involved in significantly fewer atfault crashes, providing some preliminary evidence that perceptual training can reduce crash risk.

\section{Eye Scanning Training}

Recent experimental work has observed that intersection crashes, of which older adults are at a greater risk, may be due to insufficient scanning of the roadway environment [37]. It has been hypothesized that seniors develop an unsafe strategy of primarily scanning regions immediately ahead of their vehicle at the cost of scanning peripheral intersection locations likely to contain potential threats. With training, older adults can scan more effectively and learn to allocate their attention similar to younger adults. However, more work is necessary to fully understand the extent to which greater attention to these regions benefit older driver safety.

\section{Physical Training}

Unsafe driving can also be a consequence of difficulties experienced while physically interacting with the control elements of an automobile. Some interventions have focused on improving physical functioning (specifically flexibility/range of motion) to improve driving performance. For example, Marottoli et al. [38] observed improved on-road performance following a 12-week exercise intervention. Evidence that such interventions result in fewer crashes, however, is lacking. Like all interventions discussed, large-scale clinical trials that record crash rates after the intervention compared to a suitable control group would contribute substantially to our understanding of the effectiveness of these interventions in reducing injuries, fatalities, and property damage.

\section{Conclusion}

Although older adults are at greater risk of being killed or injured in a crash due to declining perceptual, cognitive, and motor abilities, appropriate countermeasures

and training may reduce these risks. In other words, we may be able to change the roadway to better match the capabilities of older drivers or improve the abilities and strategies of older drivers to better match the demands of the driving task. Maximal safety will be achieved by pursuing both approaches.

We have largely focused on older adult drivers, but important research has been done and should continue with respect to improving the safety of all aging roadusers including pedestrians and cyclists. Like older drivers, older pedestrians are also at greater risk compared to their younger counterparts. Since physical exercise is beneficial to health and cognition in old age, infrastructure encouraging activity and protecting pedestrians and cyclists from harm may be especially beneficial to older adults.

We focused primarily on the driving difficulties of older adults and training and countermeasures to reduce these difficulties, and thus were not able to discuss all of the important issues related to aging road user safety. As mentioned previously, driving cessation is another critical issue, and related to this is the assessment of driver fitness. We primarily discussed ways to change the roadway to improve older driver safety, but changes to the vehicle itself may also play an important role. Technology can supplement the abilities of older adults [39], and features such as back-up cameras, collision warning systems, and someday in the near future autonomous or semiautonomous vehicles may help to reduce the greater risk experienced by older adult drivers.

\section{Acknowledgements}

We wish to acknowledge the National Institute on Aging (NIA 3 PO1 AG017211, Project CREATE III - Center for Research and Education on Aging and Technology Enhancement) and the Florida Department of Transportation for supporting our work on aging and transportation. We regret that due to space and reference constraints we were unable to discuss all of the important work that has been done in the area of aging roaduser safety.

References

Gerontology 2014;60:90-96 DOI: $10.1159 / 000354212$
Tefft BC: Risks older drivers pose to themselves and to other road users. J Safety Res 2008;39:577-582.

2 Edwards JD, Lunsman M, Perkins M, Rebok GW, Roth DL: Driving cessation and health trajectories in older adults. J Gerontol A Biol Sci Med Sci 2009;64:1290-1295. 
3 Staplin L, Lococo KH, Martell C, Stutts J: Taxonomy of older driver behaviors and crash risk, 2012. DOT HS 811 468A. www.nhtsa. gov/staticfiles/nti/pdf/811468a.pdf (accessed 1/6/2013).

4 Fisk AD, Rogers WA, Charness N, Czaja SJ, Sharit J: Designing for Older Adults: Principles and Creative Human Factors Approaches, ed 2. Boca Raton, CRC Press, 2009.

5 Owsley C, McGwin G Jr: Vision impairment and driving. Surv Ophthalmol 1999;43:535550.

6 Andersen GJ: Aging and vision: changes in function and performance from optics to perception. Wiley Interdiscip Rev Cogn Sci 2012; 3:403-410.

7 Li-Korotky HS: Age-related hearing loss: quality of care for quality of life. Gerontologist 2012;52:265-271.

-8 Ivers RQ, Mitchell P, Cumming RG: Sensory impairment and driving: the Blue Mountains Eye Study. Am J Public Health 1999;89:85-87.

-9 Green KA, McGwin G, Owsley C: Associations between visual, hearing, and dual sensory impairments and history of motor vehicle collision involvement of older drivers. J Am Geriatr Soc 2013;61:252-257.

10 Bédard M, Leonard E, McAuliffe J, Weaver B, Gibbons C, Dubois S: Visual attention and older drivers: the contribution of inhibition of return to safe driving. Exp Aging Res 2006;32: 119-135.

$\$ 11$ Owsley C, Ball K, Sloane ME, Roenker DL, Bruni JR: Visual/cognitive correlates of vehicle accidents in older drivers. Psychol Aging 1991;6:403-415.

12 Ball K, Owsley C: Identifying correlates of accident involvement for the older driver. Hum Factors 1991;33:583-595.

$\checkmark 13$ Salthouse TA: The processing-speed theory of adult age differences in cognition. Psychol Rev 1996;103:403-428.

14 Jastrzembski TS, Charness N: The Model Human Processor and the older adult: parameter estimation and validation within a mobile phone task. J Exp Psychol Appl 2007;13:224248.

15 Machlin S, Cohen J, Beauregard K: Health Care Expenses for Adults with Chronic Conditions, 2005. Statistical Brief No. 203. Rockville, Agency for Healthcare Research and Quality, 2008. http://www.meps.ahrq.gov/ mepsweb/data_files/publications/st203/ stat203.pdf (accessed 9/11/09).
16 Diller E, Cook L, Leonard D, Reading J, Dean JM, Vernon D: Evaluating drivers licensed with medical conditions in Utah, 1992-1996. DOT HS 809 023. Washington, National Highway Traffic Safety Administration, 1999.

7 McGwin G, Sims RV, Pulley L, Roseman JM: Diabetes and automobile crashes in the elderly. A population-based case-control study. Diabetes Care 1999;22:220-227.

18 Preusser DF, Williams AF, Ferguson SA, Ulmer RG, Weinstein HB: Fatal crash risk for older drivers at intersections. Accid Anal Prev 1998;30:151-159.

19 Scialfa CT, Guzy LT, Leibowitz HW, Garvey PM, Tyrrell RA: Age differences in estimating vehicle velocity. Psychol Aging 1991;6:60-66.

20 Anderson GJ, Enriquez A: Aging and the detection of observer and moving object collisions. Psychol Aging 2006;21:74-85.

21 Owens DA, Tyrrell RA: Effects of luminance, blur, and age on nighttime visual guidance: a test of the selective degradation hypothesis. J Exp Psychol Appl 1999;5:115.

22 Caird JK, Edwards CJ, Creaser JL, Horrey WJ: Older driver failures of attention at intersections: using change blindness methods to assess turn decision accuracy. Hum Factors 2005; 47:235-249.

23 Dewar R, Kline D, Scheiber F, Swanson A: Symbol Signing Design for Older Drivers. Publication No. FHWA-RD-94-069. Washington, US Department of Transportation, Federal Highway Administration, 1997.

24 Staplin L, Lococo K, Byington S, Harkey D: Guidelines and Recommendations to Accommodate Older Drivers and Pedestrians. Publication No. FHWA-RD-01-051. Washington, US Department of Transportation, Federal Highway Administration, 2001.

25 Staplin L, Harkey D, Lococo K, Tarawneh M: Intersection Geometric Design and Operational Guidelines for Older Drivers and Pedestrians, Volume I: Final Report. Publication No. FHWA-RD-96-132. Washington, US Department of Transportation, Federal Highway Administration, 1997.

26 Easa S, Reed M, Russo F, Dabbour E, Mehmood E, Curtis K: Effect of increasing road light luminance on night driving performance of older adults. Int J Appl Sci Eng Technol 2010:6:41-48.

27 Gross F, Lefler N, Lyon C, Eccles K: Safety effectiveness of advanced street name signs. Transp Res Rec 2010;2185:55-62.
28 Guerrier JH, Fu S-H. Elder Roadway User Program Test Sections and Effectiveness Study: Final Report, Task 4, Subtask One (University of Miami Project No. 669535). Miami, University of Miami, 2002.

29 Card SK, Moran TP, Newell A: The Psychology of Human-Computer Interaction. Hillsdale, Lawrence Erlbaum Associates, 1983.

30 Caird JK, Chisholm SL, Edwards CJ, Creaser JI: The effect of yellow light onset time on older and younger drivers' perception response time (PRT) and intersection behavior. Transp Res F Traffic Psychol Behav 2007; 10:383-396.

31 Owsley C, McGwin G, Phillips JM, McNeal SF, Stalvey BT: Impact of an educational program on the safety of high-risk, visually impaired, older drivers. Am J Prev Med 2004;26: 222-229.

32 Janke MK: Mature driver improvement program in California. Transp Res Rec 1994; 1438:77-83.

33 Bédard M: The combination of two training approaches to improve older adults' driving safety. Traffic Inj Prev 2008;9:70-76.

34 Marottoli RA, Van Ness PH, Araujo KL, Iannone LP, Acampora D, Charpentier P, Peduzzi P: A randomized trial of an education program to enhance older driver performance. J Gerontol A Biol Sci Med Sci 2007;62: 1113-1119.

35 Roenker DL, Cissell GM, Ball KK, Wadley VG, Edwards JD: Speed-of-processing and driving simulator training result in improved driving performance. Hum Factors 2003;45: 218-233.

- 36 Ball K, Edwards JD, Ross LA, McGwin GJ: Cognitive training decreases motor vehicle collision involvement of older drivers. J Am Geriatr Soc 2010;58:2107-2113.

37 Pollatsek A, Romoser MRE, Fisher DL: Identifying and remediating failures of selective attention in older drivers. Curr Dir Psychol Sci 2012;21:3-7.

38 Marottoli RA, Araujo KL, Iannone LP, Acampora D, Margaret Gottschalk PT, MS, Charpentier P: A randomized trial of a physical conditioning program to enhance the driving performance of older persons. J Gen Intern Med 2007;22:590-597.

39 Charness N, Boot WR: Aging and information technology use: potential and barriers. Curr Dir Psychol Sci 2009;18:253-258. 